\title{
Associations between dietary patterns and age at natural menopause in the UK Women's Cohort Study
}

\author{
Y. Dunneram ${ }^{1}$, D.C. Greenwood ${ }^{2}$ and J.E. Cade ${ }^{1}$ \\ ${ }^{1}$ Nutritional Epidemiology Group, School of Food Science \& Nutrition and ${ }^{2}$ Division of Biostatistics, University of \\ Leeds, Leeds LS2 9JT, UK
}

Previous studies have demonstrated an association between a later menopause and reduced risk of cardiovascular diseases and osteoporosis, and a higher risk for endometrial, ovarian and breast cancer ${ }^{(1)}$. It is hypothesized that diet can influence the timing of natural menopause ${ }^{(2)}$. Yet, evidence linking diet and age at natural menopause is still sparse and inconsistent. This study aimed to investigate the prospective association between dietary patterns derived from two different methods and age at natural menopause.

Menopausal status was reported at two time points 4 years apart in the UK Women's Cohort Study. Diet of participants were measured using a 217-item food frequency questionnaire at baseline. Principal component analysis (PCA) and reduced ranked regression (RRR) were used to derive dietary patterns for 13,916 women. Cox proportional hazards regression models were used to estimate hazard ratios (HR) and $95 \%$ confidence intervals (CI) for each pattern in relation to increasing age at natural menopause at follow-up, adjusting for potential confounders. Five patterns were identified from the PCA, which we labelled: 'vegetables and legumes', 'animal proteins', 'fruits', 'fats and sweets' and 'low-fat products'. Three patterns were derived from RRR: 'sweets, pastries and puddings', 'low-fat dairy and meat', and 'red meat and processed meat'. Women who scored higher on the 'animal proteins' pattern were $6 \%$ less likely to have gone through a natural menopause (HR: $0.94,95 \%$ CI: 0.90 to 0.97 ) compared to those who scored lower. The 'red meat and processed meat' pattern predicted a $7 \%$ higher risk for a later natural menopause (HR: $0.93,95 \%$ CI: 0.87 to 1.00). No evidence of an association was observed between the other dietary patterns and incidence of being naturally menopausal.

\begin{tabular}{|c|c|c|c|c|c|c|}
\hline \multirow[b]{2}{*}{ Dietary patterns } & \multicolumn{3}{|c|}{ Crude $^{\dagger}$} & \multicolumn{3}{|c|}{ Model 1* } \\
\hline & $\overline{\mathrm{HR}}$ & $95 \% \mathrm{CI}$ & $P$ & $\overline{\mathrm{HR}}$ & $95 \% \mathrm{CI}$ & $P$ \\
\hline \multicolumn{7}{|l|}{ Principal component analysis } \\
\hline Vegetables and legumes & 0.99 & 0.96 to 1.01 & 0.34 & 1.00 & 0.97 to 1.03 & 0.92 \\
\hline Animal proteins & 0.95 & 0.91 to 0.98 & $<0.01$ & 0.94 & 0.90 to 0.97 & $<0.01$ \\
\hline Fruits & 1.05 & 1.01 to 1.09 & 0.02 & 1.04 & 0.99 to 1.08 & 0.12 \\
\hline Fats and sweets & 1.00 & 0.95 to 1.05 & 0.94 & 1.00 & 0.94 to 1.07 & 0.98 \\
\hline Low-fat products & 0.99 & 0.95 to 1.04 & 0.77 & 0.97 & 0.92 to 1.03 & 0.30 \\
\hline \multicolumn{7}{|l|}{ Reduced ranked regression } \\
\hline Sweets, pastries and puddings & 0.95 & 0.89 to 1.01 & 0.12 & 0.96 & 0.88 to 1.04 & 0.28 \\
\hline Low-fat dairy and meat & 0.96 & 0.89 to 1.02 & 0.17 & 0.97 & 0.90 to 1.04 & 0.38 \\
\hline Red meat and processed meat & 0.95 & 0.89 to 1.01 & 0.11 & 0.93 & 0.87 to 1.00 & 0.05 \\
\hline
\end{tabular}

$\dagger$ Unadjusted model; *Adjusted for smoking status, alcohol consumption, physical activity, and social class.

This is the first study ever investigating the associations between dietary patterns and age at natural menopause. Both PCA and RRR are useful in deriving dietary patterns which can influence the onset of natural menopause. These findings will contribute to an improved understanding of the timing of natural menopause in relation to diet, which may also have implications associated with longer term health outcomes in post-menopausal women.

This work was supported by the Commonwealth Scholarships, UK.

1. Gold EB (2011) Obstet Gynecol Clin North Am 38, 425-440.

2. Nagata C, Takatsuka N, Inaba S et al. (1998) Maturitas 29, 105-113. 